\title{
Supplementary material: Efficient model comparison techniques for models requiring large scale data augmentation
}

\author{
Panayiota Touloupou, Naif Alzahrani, Peter Neal, \\ Simon Spencer and Trevelyan J. McKinley
}

\section{Linear mixed model}

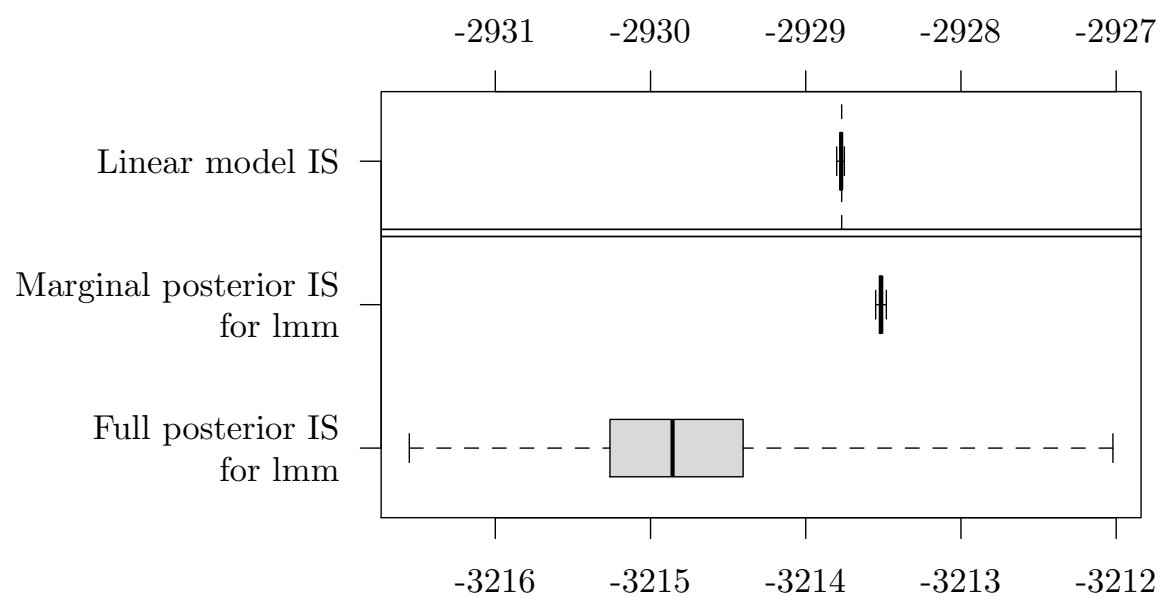

Log marginal likelihood

Figure 1: Variation of the log marginal likelihood estimates for the linear model and linear mixed model $(\mathrm{lmm})$ over 50 replicates. Simulated data contain 500 clusters of size 3 . For the $1 \mathrm{~mm}$, in the full posterior approach the whole parameter vector (including random effects) was approximated in the importance proposal; in the marginal posterior approach the random effects were left out, and drawn from their full conditional distribution. A dashed vertical line indicates the true log marginal likelihood for the linear model.

In this section we present some additional results for the linear mixed model. In particular we investigate what happens when the number of random effects $m$, (the dimension of the missing data) increases. We produced simu- 


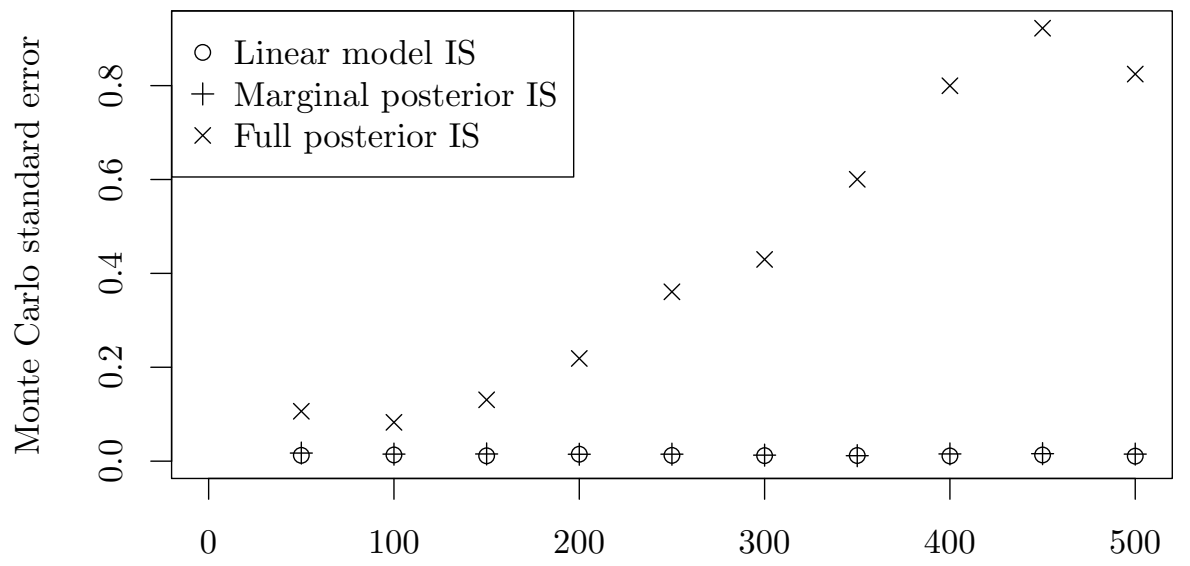

Number of clusters, $m$

Figure 2: Monte Carlo standard error of the log marginal likelihood estimates for the linear model and linear mixed model ( $\mathrm{lmm}$ ) over 50 replicates. Simulated datasets contain between 50 and 500 clusters of size 3. For the $\mathrm{lmm}$, in the full posterior approach the whole parameter vector (including random effects) was approximated in the importance proposal; in the marginal posterior approach the random effects were left out, and drawn from their full conditional distribution.

lated datasets for $m$ between 50 and 500 and followed the same procedures for estimating the log marginal likelihood described in the main text.

Figure 1 shows the variation in the log marginal likelihoods for the linear model importance sampler and the linear mixed model $(\mathrm{lmm})$ with the full posterior importance sampler and marginal posterior importance sampler. Figure 2 shows how the Monte Carlo standard errors increase as the number of clusters $m$ is increased from 50 to 500 . The marginal posterior importance sampling estimator doesn't become less precise as the number of random effects increases, in contrast to the full posterior importance sampler.

\section{Final outcome epidemic data}

In this Section, we show how the approach taken in Neal and Kypraios (2015), Section 3, can be adapted to compute the exact marginal likelihood for household Reed-Frost epidemics.

Suppose that we observe an epidemic of size $k$ in a household of size $h$ then 
the recursive equations of Ball et al. (1997), (3.12) can be used to compute the probability of this epidemic given parameters $p_{G}$ and $p_{L}$ for the probability of being infected globally from the community and the within household probability of infection, respectively. However, to enable us to compute exactly the marginal likelihood data augmentation is required defining how the epidemic spread throughout the household. Specifically, we construct the epidemic on a generational basis starting with those individuals who were infected globally. Let $i_{0}$ denote the total number of individuals infected globally in the household, and label these individuals generation 0 . For $j=1,2, \ldots$, let $i_{j}$ denote the total number of individuals infected in generation $j$ by the $i_{j-1}$ individuals in generation $j-1$ and let $m_{j}$ denote the total number of infectious contacts made by the $i_{j-1}$ individuals in generation $j-1$ who are susceptible at the start of generation $j$. Note that $m_{j} \geq i_{j}$. The epidemic finishes at generation $J$, if $m_{J+1}=i_{J+1}=0$ and no infections in the household if $i_{0}=0$. Let $\left\{i_{0},\left(i_{1}, m_{1}\right), \ldots,\left(i_{J}, m_{J}\right)\right\}$ denote the course of the epidemic with $k=\sum_{j=0}^{J} i_{j}$, then it is straightforward to show that

$$
\begin{aligned}
& P\left(i_{0},\left(i_{1}, m_{1}\right), \ldots,\left(i_{J}, m_{J}\right) \mid p_{L}, p_{G}\right) \\
= & \left(\begin{array}{c}
h \\
i_{0}
\end{array}\right) p_{G}^{i_{0}}\left(1-p_{G}\right)^{h-i_{0}} \times \prod_{j=1}^{J+1} C\left(i_{j-1}, s_{j-1}, i_{j}, m_{j}\right) p_{L}^{m_{j}}\left(1-p_{L}\right)^{s_{j-1} i_{j-1}-m_{j}}
\end{aligned}
$$

where $s_{-1}=h$ and for $j=0,1, \ldots, s_{j}=s_{j-1}-i_{j}$ and $C\left(i_{j-1}, s_{j-1}, i_{j}, m_{j}\right)$ is the total number of ways in which $i_{j}$ individuals out of $s_{j-1}$ individuals can be infected by $i_{j-1}$ individuals making $m_{j}$ infectious contacts. The computation of $\tilde{C}_{\mathbf{i}, \mathbf{m}}=\left(\begin{array}{c}h \\ i_{0}\end{array}\right) \prod_{j=1}^{J+1} C\left(i_{j-1}, s_{j-1}, i_{j}, m_{j}\right)$ is given in the table in Appendix A of Neal and Kypraios (2015) for $k \leq 4$. Let $\tilde{A}_{\mathbf{i}, \mathbf{m}}=\sum_{i=1}^{J+1} m_{j}$ and $\tilde{B}_{\mathbf{i}, \mathbf{m}}=\sum_{i=1}^{J+1}\left(s_{j-1} i_{j-1}-m_{j}\right)$. Then

$P\left(i_{0},\left(i_{1}, m_{1}\right), \ldots,\left(i_{J}, m_{J}\right) \mid p_{L}, p_{G}\right)=\tilde{C}_{\mathbf{i}, \mathbf{m}} p_{G}^{i_{0}}\left(1-p_{G}\right)^{h-i_{0}} p_{L}^{\tilde{A}_{\mathbf{i}, \mathbf{m}}}\left(1-p_{L}\right)^{\tilde{B}_{\mathbf{i}, \mathbf{m}}}$,

and it is this representation of the epidemic which we can exploit in computing the marginal likelihood exactly.

For households where nobody or only one person becomes infected there is only one possible $(\mathbf{i}, \mathbf{m})$ consistent with the within household epidemic. This number rapidly grows with there being $2,5,13$ and 33 possibilities for $(\mathbf{i}, \mathbf{m})$ in households with $2,3,4$ and 5 infected individuals in, respectively. Therefore we augment the observed data $\mathbf{x}=\left(x_{h k}\right)$, where $x_{h k}$ is the number of households of size $h$ with $k$ infected by $\mathbf{y}=\left(y_{h k l}\right)$, where $y_{h k l}$ is the number of households of size $h$ with $k$ infected and the within household infection 
is given by the $l^{\text {th }}$ such infection path. For $k=0,1,2,3,4,5$, the number of possibilities, $L_{k}$, for $l$ are 1,1,2,5,13 and 33, respectively. Using (2) it is straightforward to show that for $\mathbf{y}$ consistent with $\mathbf{x}$,

$$
\begin{aligned}
& \pi\left(\mathbf{x}, \mathbf{y} \mid p_{L}, p_{G}\right) \\
& =\prod_{h=1}^{h \max } \prod_{k=0}^{h} \frac{x_{h k} !}{\prod_{l=1}^{L_{j}} y_{h k l} !} \\
& \times \prod_{h=1}^{h \max } \prod_{k=0}^{h} \prod_{l=1}^{L_{k}}\left\{\hat{C}_{h k l} p_{G}^{\hat{D}_{h k l}}\left(1-p_{G}\right)^{\hat{E}_{h k l}} p_{L}^{\hat{A}_{h k l}}\left(1-p_{L}\right)^{\hat{B}_{h k l}}\right\}^{y_{h k l}} \\
& =C^{*}(\mathbf{x}, \mathbf{y}) p_{G}^{D^{*}(\mathbf{x}, \mathbf{y})}\left(1-p_{G}\right)^{E^{*}(\mathbf{x}, \mathbf{y})} p_{L}^{A^{*}(\mathbf{x}, \mathbf{y})}\left(1-p_{L}\right)^{B^{*}(\mathbf{x}, \mathbf{y})},
\end{aligned}
$$

where for $X=A, B, C, D, E, \hat{X}_{h k l}$ is the corresponding quantity for the infectious path under consideration and for $X=A, B, D, E, X^{*}(\mathbf{x}, \mathbf{y})=$ $\sum_{h=1}^{h \max } \sum_{k=0}^{h} \sum_{l=1}^{L_{k}} y_{h k l} \hat{X}_{h k l}$. Note that $C^{*}(\mathbf{x}, \mathbf{y})$ is the overall multiplicative constant. Therefore since $\pi\left(p_{G}\right)=\pi\left(p_{L}\right)=1\left(0<p_{G}, p_{L}<1\right) \pi(\mathbf{x}, \mathbf{y})$ can be obtained by integrating (3) with respect to $p_{G}$ and $p_{L}$ giving

$\pi(\mathbf{x}, \mathbf{y})=C^{*}(\mathbf{x}, \mathbf{y}) \frac{D^{*}(\mathbf{x}, \mathbf{y}) ! E^{*}(\mathbf{x}, \mathbf{y}) !}{\left(D^{*}(\mathbf{x}, \mathbf{y})+E^{*}(\mathbf{x}, \mathbf{y})+1\right) !} \frac{A^{*}(\mathbf{x}, \mathbf{y}) ! B^{*}(\mathbf{x}, \mathbf{y}) !}{\left(A^{*}(\mathbf{x}, \mathbf{y})+B^{*}(\mathbf{x}, \mathbf{y})+1\right) !}$.

This gives the posterior probability of observing the augmented outcome $\mathbf{y}$. To obtain the marginal likelihood, $\pi(\mathbf{x})$, we need to sum over $\mathbf{y} \in \mathcal{N}(\mathbf{x})$, where $\mathcal{N}(\mathbf{x})$ denotes the set of all possible $\mathbf{y}$ consistent with $\mathbf{x}$. Thus

$$
\pi(\mathbf{x})=\sum_{\mathbf{y} \in \mathcal{N}(\mathbf{x})} \frac{C^{*}(\mathbf{x}, \mathbf{y}) A^{*}(\mathbf{x}, \mathbf{y}) ! B^{*}(\mathbf{x}, \mathbf{y}) ! D^{*}(\mathbf{x}, \mathbf{y}) ! E^{*}(\mathbf{x}, \mathbf{y}) !}{\left(D^{*}(\mathbf{x}, \mathbf{y})+E^{*}(\mathbf{x}, \mathbf{y})+1\right) !\left(A^{*}(\mathbf{x}, \mathbf{y})+B^{*}(\mathbf{x}, \mathbf{y})+1\right) !}
$$

For the Seattle influenza A and influenza B data sets, $|\mathcal{N}(\mathbf{x})|$ is 13860 and 157500 , respectively, but for the Tecumseh data $|\mathcal{N}(\mathbf{x})|$ is $2.820 \times 10^{20}$, see Neal and Kypraios (2015), Table 1.

\section{Longitudinal epidemic model}

\subsection{Marginal likelihood estimation}

In this section we briefly overview alternative techniques for estimating the likelihood. However first we give the full list of proposals distributions based on a fitted multivariate normal distribution with mean $\boldsymbol{\mu}$ and covariance matrix $\boldsymbol{\Sigma}$ used in the importance sampling. These are 
1) $\mathrm{IS}_{N_{1}}: q(\boldsymbol{\theta})=N(\boldsymbol{\theta} ; \boldsymbol{\mu}, \boldsymbol{\Sigma})$,

2) $\operatorname{IS}_{N_{2}}: q(\boldsymbol{\theta})=N(\boldsymbol{\theta} ; \boldsymbol{\mu}, 2 \boldsymbol{\Sigma})$,

3) $\operatorname{IS}_{N_{3}}: q(\boldsymbol{\theta})=N(\boldsymbol{\theta} ; \boldsymbol{\mu}, 3 \boldsymbol{\Sigma})$,

4) $\mathrm{IS}_{\text {mix }}: q(\boldsymbol{\theta})=0.95 \times N(\boldsymbol{\theta} ; \boldsymbol{\mu}, \boldsymbol{\Sigma})+0.05 \times \pi(\boldsymbol{\theta})$,

5) $\mathrm{IS}_{t_{4}}: q(\boldsymbol{\theta})=t_{4}(\boldsymbol{\theta} ; \boldsymbol{\mu}, \boldsymbol{\Sigma})$,

6) $\mathrm{IS}_{t_{6}}: q(\boldsymbol{\theta})=t_{6}(\boldsymbol{\theta} ; \boldsymbol{\mu}, \boldsymbol{\Sigma})$,

7) $\mathrm{IS}_{t_{8}}: q(\boldsymbol{\theta})=t_{8}(\boldsymbol{\theta} ; \boldsymbol{\mu}, \boldsymbol{\Sigma})$,

where $t_{d}(\boldsymbol{\theta}, \boldsymbol{\mu}, \boldsymbol{\Sigma})$ is the density of the multivariate Student's $t$ distribution with $d$ degrees of freedom, mean $\boldsymbol{\mu}$ and covariance matrix $\frac{d}{d-2} \boldsymbol{\Sigma}$ (if $d>2$ ).

\subsubsection{Marginal likelihood estimation via the harmonic mean}

The harmonic mean (HM) estimator (Newton and Raftery, 1994) can be computed directly from MCMC output, which has lead to its widespread use. When data augmentation is used, the parameter vector comprises latent variable $\mathbf{y}$ as well as the model parameters $\boldsymbol{\theta}$. The marginal likelihood $\pi(\mathbf{x})$ can be approximated by the sample harmonic mean of the likelihoods,

$$
\hat{P}_{H M}(\mathbf{x})=\left[\frac{1}{N} \sum_{i=1}^{N} \frac{1}{P\left(\mathbf{x} \mid \mathbf{y}_{i}, \boldsymbol{\theta}_{i}\right)}\right]^{-1}
$$

based on $N$ draws $\left(\mathbf{y}_{1}, \boldsymbol{\theta}_{1}\right),\left(\mathbf{y}_{2}, \boldsymbol{\theta}_{2}\right), \ldots,\left(\mathbf{y}_{N}, \boldsymbol{\theta}_{N}\right)$ from the joint posterior $\pi(\mathbf{y}, \boldsymbol{\theta} \mid \mathbf{x})$. Although asymptotically consistent, the harmonic mean estimator is known to exhibit large or even infinite variance for some models.

\subsubsection{Marginal likelihood estimation via bridge sampling}

Meng and Wong (1996) introduced the bridge sampling (BS) as a samplebased technique for computing ratios of normalising constants. The key identity for bridge sampling is given by,

$$
\pi(\mathbf{x})=\frac{\mathrm{E}_{q(\boldsymbol{\theta})}\left\{\alpha(\boldsymbol{\theta}) \pi^{*}(\boldsymbol{\theta} \mid \mathbf{x})\right\}}{\mathrm{E}_{\pi(\boldsymbol{\theta} \mid \mathbf{x})}\{\alpha(\boldsymbol{\theta}) q(\boldsymbol{\theta})\}}
$$

for an arbitrary function $\alpha$, where $\pi^{*}(\boldsymbol{\theta} \mid \mathbf{x})=\pi(\boldsymbol{\theta}) \pi(\mathbf{x} \mid \boldsymbol{\theta})$ is the unnormalised posterior and $q$ is a normalised density. Meng and Wong (1996) show that 
the optimal choice for $\alpha$ leads to the following iterative procedure to obtain the bridge sampling estimator of the marginal likelihood,

$$
\widehat{P}_{\mathrm{BS}}^{(t)}(\mathbf{x})=\widehat{P}_{\mathrm{BS}}^{(t-1)}(\mathbf{x}) \frac{\frac{1}{L} \sum_{l=1}^{L} \frac{\hat{\pi}^{(t-1)}\left(\tilde{\boldsymbol{\theta}}_{l} \mid \mathbf{x}\right)}{L q\left(\tilde{\boldsymbol{\theta}}_{l}\right)+M \hat{\pi}^{(t-1)}\left(\tilde{\boldsymbol{\theta}}_{l} \mid \mathbf{x}\right)}}{\frac{1}{M} \sum_{m=1}^{M} \frac{q\left(\hat{\boldsymbol{\theta}}_{m}\right)}{L q\left(\hat{\boldsymbol{\theta}}_{m}\right)+M \hat{\pi}^{(t-1)}\left(\hat{\boldsymbol{\theta}}_{m} \mid \mathbf{x}\right)}},
$$

where $\hat{\pi}^{(t-1)}\left(\boldsymbol{\theta}_{m} \mid \mathbf{x}\right)=\pi^{*}(\boldsymbol{\theta} \mid \mathbf{x}) / \widehat{P}_{\mathrm{BS}}^{(t-1)}(\mathbf{x})$, using MCMC i.i.d. draws $\hat{\boldsymbol{\theta}}_{m}, m=$ $1,2, \ldots, M$ from the posterior $\pi(\boldsymbol{\theta} \mid \mathbf{x})$ and $\tilde{\boldsymbol{\theta}}_{l}, l=1,2, \ldots, L$ from the importance sampling density $q(\boldsymbol{\theta})$.

With missing data the estimator can be written as,

$$
\widehat{P}_{\mathrm{BS}}^{(t)}(\mathbf{x})=\widehat{P}_{\mathrm{BS}}^{(t-1)}(\mathbf{x}) \frac{\frac{1}{L} \sum_{l=1}^{L} \frac{1}{\frac{L q\left(\tilde{\boldsymbol{\theta}}_{l}\right) \pi\left(\tilde{\mathbf{y}}_{l} \mid \mathbf{x}, \tilde{\boldsymbol{\theta}}_{l}\right) \widehat{P}_{\mathrm{BS}}^{(t-1)}(\mathbf{x})}{\pi\left(\mathbf{x}, \tilde{\mathbf{y}}_{l} \mid \tilde{\boldsymbol{\theta}}_{l}\right) \pi\left(\tilde{\boldsymbol{\theta}}_{l}\right)}+M}}{\frac{1}{M} \sum_{m=1}^{M} \frac{1}{L+\frac{M \pi\left(\mathbf{x}, \hat{\mathbf{y}}_{m} \mid \hat{\boldsymbol{\theta}}_{m}\right) \pi\left(\hat{\boldsymbol{\theta}}_{m}\right)}{q\left(\hat{\boldsymbol{\theta}}_{m}\right) \pi\left(\hat{\mathbf{y}}_{m} \mid \mathbf{x}, \hat{\boldsymbol{\theta}}_{m}\right) \widehat{P}_{\mathrm{BS}}^{(t-1)}(\mathbf{x})}}} .
$$

The proposed importance sampling estimator, which is a special case of the general bridge sampling estimator, is used as starting value $\widehat{P}_{\mathrm{BS}}^{(0)}(\mathbf{x})$. In practise, iterative application of (7) is very fast.

\subsubsection{Marginal likelihood estimation via Chib's method}

Chib's method for estimating the marginal likelihood (Chib, 1995; Chib and Jeliazkov, 2001) is based on the observation that,

$$
\pi(\mathbf{x})=\frac{\pi(\mathbf{x} \mid \boldsymbol{\theta}) \pi(\boldsymbol{\theta})}{\pi(\boldsymbol{\theta} \mid \mathbf{x})}
$$

for all $\boldsymbol{\theta}$ in the support of the posterior. For fixed $\boldsymbol{\theta}=\boldsymbol{\theta}^{*}$ the $\log$ marginal likelihood can be estimated by

$$
\log \widehat{P}_{\mathrm{Chib}}(\mathbf{x})=\log \pi\left(\mathbf{x} \mid \boldsymbol{\theta}^{*}\right)+\log \pi\left(\boldsymbol{\theta}^{*}\right)-\log \widehat{\pi}\left(\boldsymbol{\theta}^{*} \mid \mathbf{x}\right)
$$

The prior and likelihood can be easily evaluated at $\boldsymbol{\theta}^{*}$. The posterior density is estimated by breaking the parameter vector into appropriate blocks. For blocks that can be updated using a Gibb's step the normalising constant of the full conditional distribution is known and so MCMC samples of the 
remaining blocks can be used to estimate the normalising constant of the posterior. For components of $\boldsymbol{\theta}$ that are updated using Metropolis-Hastings, the required normalising constant can be estimated from the acceptance probabilities of a jump from the current state to $\boldsymbol{\theta}^{*}$.

For the model described in Section 5.1 we decompose the parameter vector into $\left(\mathbf{y}, \boldsymbol{\theta}_{1}, \boldsymbol{\theta}_{2}, \boldsymbol{\theta}_{3}\right)$, where $\boldsymbol{\theta}_{1}=\left(k_{1}, k_{2}, \beta_{11}, \beta_{12}, \beta_{21}, \beta_{22}, \mu_{1}, \mu_{2}, w\right), \boldsymbol{\theta}_{2}=\pi_{1}$ and $\boldsymbol{\theta}_{3}=\pi_{2}$. The posterior density is then factorised as

$$
\pi\left(\mathbf{y}^{*}, \boldsymbol{\theta}^{*} \mid \mathbf{x}\right)=\pi\left(\mathbf{y}^{*} \mid \mathbf{x}\right) \pi\left(\boldsymbol{\theta}_{1}^{*} \mid \mathbf{x}, \mathbf{y}^{*}\right) \pi\left(\boldsymbol{\theta}_{2}^{*} \mid \mathbf{x}, \mathbf{y}^{*}, \boldsymbol{\theta}_{1}^{*}\right) \pi\left(\boldsymbol{\theta}_{3}^{*} \mid \mathbf{x}, \mathbf{y}^{*}, \boldsymbol{\theta}_{1}^{*}, \boldsymbol{\theta}_{2}^{*}\right) .
$$

To calculate each term in this product, a separate MCMC chain is run in which only the unconditioned blocks of $(\mathbf{y}, \boldsymbol{\theta})$ are updated and the remaining blocks are fixed at $\boldsymbol{\theta}^{*}$.

\subsubsection{Marginal likelihood estimation via power posteriors}

The Power Posterior (PP) approach to estimating the marginal likelihood (Friel and Pettitt, 2008) uses samples from the power posterior, defined as

$$
\pi_{t}(\boldsymbol{\theta} \mid \mathbf{x}) \propto \pi(\mathbf{x} \mid \boldsymbol{\theta})^{t} \pi(\boldsymbol{\theta})
$$

where $t \in[0,1]$ is a temperature parameter. Borrowing ideas from path sampling allows the log of the marginal likelihood to be represented in terms of the thermodynamic integral

$$
\log \pi(\mathbf{x})=\int_{0}^{1} \mathrm{E}_{\boldsymbol{\theta} \mid \mathbf{x}, t}\{\log \pi(\mathbf{x} \mid \boldsymbol{\theta})\} d t
$$

where the expectation of the mean deviance is taken with respect to the power posterior at temperature $t$, where $t$ moves from 0 to 1 . The integral can be calculated numerically by discretising the temperature range as $0=t_{0}<t_{1}<\ldots<t_{n}=1$, and then the log marginal likelihood can be approximated by the trapezium rule,

$$
\log \widehat{P}_{P P}(\mathbf{x})=\sum_{i=0}^{n-1}\left(t_{i+1}-t_{i}\right) \frac{\mathrm{E}_{\boldsymbol{\theta} \mid \mathbf{x}, t_{i+1}}\{\log \pi(\mathbf{x} \mid \boldsymbol{\theta})\}+\mathrm{E}_{\boldsymbol{\theta} \mid \mathbf{x}, t_{i}}\{\log \pi(\mathbf{x} \mid \boldsymbol{\theta})\}}{2} .
$$

For each $t_{i}$, samples from the power posterior $\pi_{t_{i}}(\boldsymbol{\theta} \mid \mathbf{x})$ can be used to obtain an estimate of the required expectation $\mathrm{E}_{\boldsymbol{\theta} \mid \mathbf{x}, t_{i}}\{\log \pi(\mathbf{x} \mid \boldsymbol{\theta})\}$. Metropolis within Gibbs sampling was used to obtain samples from the power posterior at each temperature $t>0$.

The variability of the power posterior estimator depends on the chosen number and spacing of the $t_{i}$ 's. Choosing a large number of temperatures, the 
estimation of the log marginal likelihood requires considerably more computational effort. Moreover, the precision of the estimate is sensitive to the number of samples used and the mixing of the MCMC sampler.

\subsection{Simulation study}

\subsubsection{Temperatures for the power posteriors}

In Friel and Pettitt (2008) the temperatures were chosen with a geometric spacing, $t_{l}=(l / n)^{c}$, for $l=0,1, \ldots, n$, with $c>1$, which places many of the temperatures close to zero. This scheme is preferable in cases where the expected deviance has a sharp increase near zero before leveling off. However, in our case, the curve of the expected deviance is not convex (Figure 3). After some pilot analysis (not counted in the computation cost) we chose to use 20 partitions of the unit line, placing more temperatures around zero and the other sharp change.

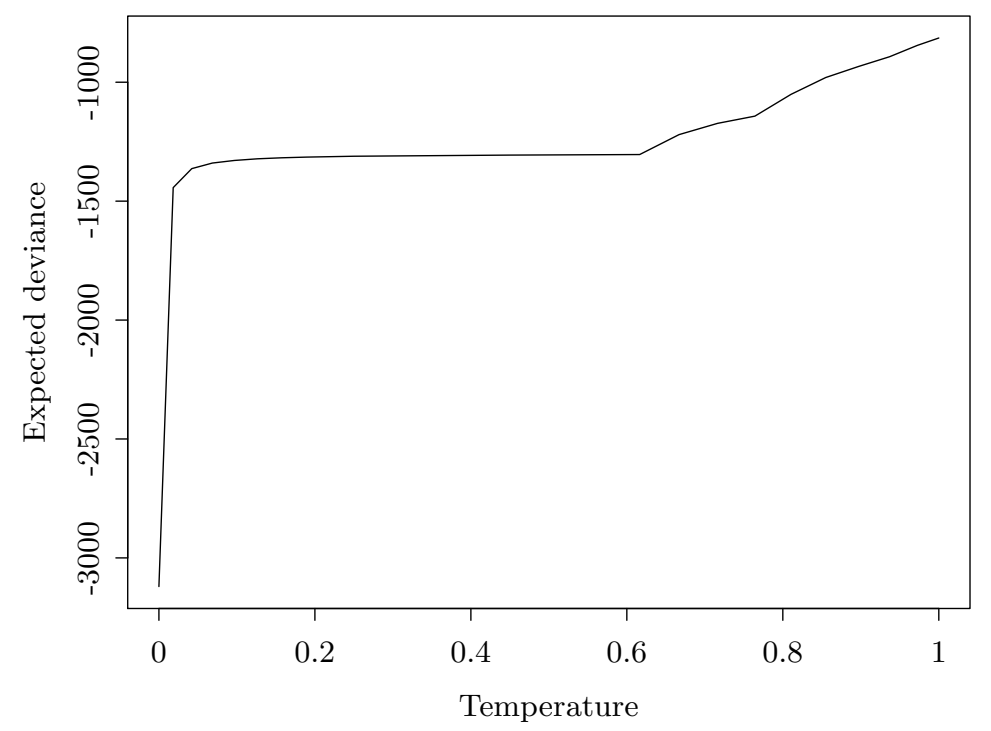

Figure 3: Expected deviance against temperature for model $\mathcal{M}_{1}$ estimated using power posteriors.

\subsubsection{Thinning for the bridge sampling}

The optimal bridge sampling estimator is constructed on the basis of having iid samples from the posterior available. Thinning can be used to reduce the 


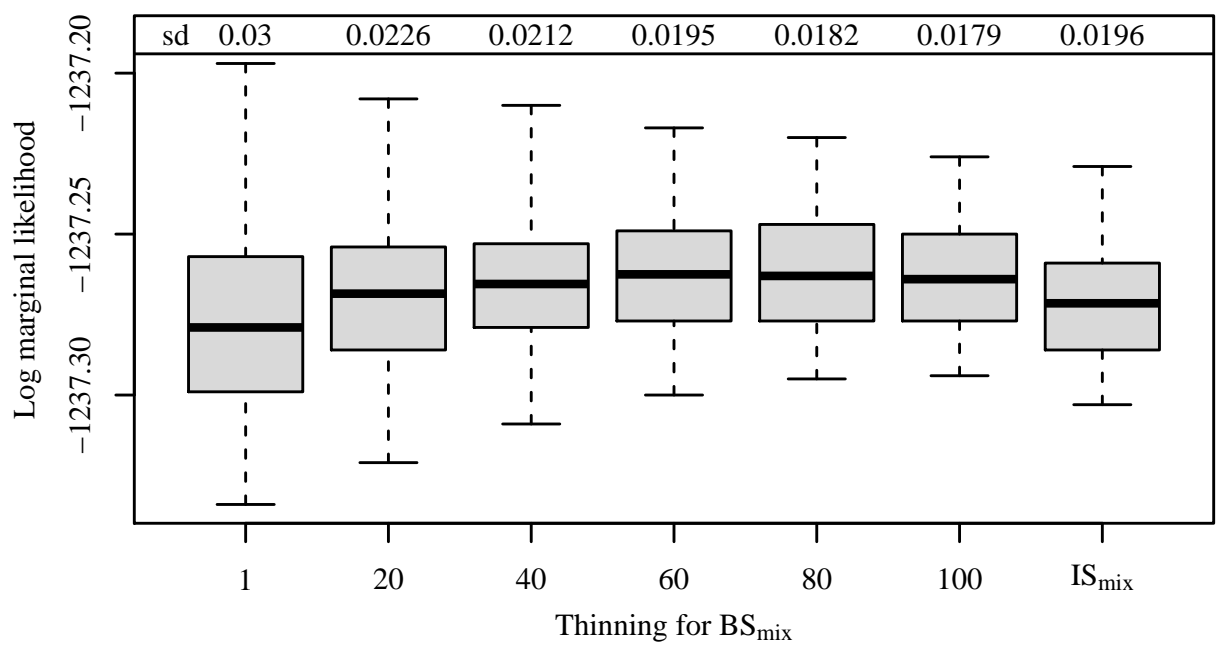

Figure 4: Effect of thinning of the MCMC samples on the Monte Carlo variance of the bridge sampling estimator, over 50 replicates.

autocorrelation in posterior samples produced using MCMC. Figure 4 shows the effect that the amount of thinning has on the bridge sampling estimator. Interestingly, quite substantial thinning is needed before the Monte Carlo variance of the bridge sampling estimator drops below that of the importance sampling estimator.

\subsubsection{Monte Carlo standard errors of log marginal likelihood es- timates}

Table 1 gives the Monte Carlo standard errors of log marginal likelihood estimates for different number of Markov chain samples. The results show that increasing the number of MC samples $n$, led to a decrease in the Monte Carlo standard errors of order $\mathcal{O}(\sqrt{n})$, indicating that the variance of the corresponding estimators is finite.

\subsection{Model comparison}

In this section we provide further details and examples of the strength of the importance sampling technique in answering model comparison questions. We begin by providing details of the reversible jump algorithm for heterogeneity in community acquisition rates. This is followed by investigations of model comparison for heterogeneity in household transmission 


\begin{tabular}{c|cc|cc|cc}
\hline \hline Method & $\begin{array}{c}\text { MC } \\
\text { samples }\end{array}$ & $\begin{array}{c}\text { MC } \\
\text { error }\end{array}$ & $\begin{array}{c}\text { MC } \\
\text { samples }\end{array}$ & $\begin{array}{c}\text { MC } \\
\text { error }\end{array}$ & $\begin{array}{c}\text { MC } \\
\text { samples }\end{array}$ & $\begin{array}{c}\text { MC } \\
\text { error }\end{array}$ \\
\hline $\mathrm{IS}_{N_{1}}$ & 10000 & 0.053 & 25000 & 0.033 & 50000 & 0.025 \\
$\mathrm{IS}_{N_{2}}$ & 10000 & 0.064 & 25000 & 0.036 & 50000 & 0.025 \\
$\mathrm{IS}_{N_{3}}$ & 10000 & 0.107 & 25000 & 0.061 & 50000 & 0.042 \\
$\mathrm{IS}_{t_{4}}$ & 10000 & 0.037 & 25000 & 0.025 & 50000 & 0.020 \\
$\mathrm{IS}_{t_{6}}$ & 10000 & 0.064 & 25000 & 0.034 & 50000 & 0.023 \\
$\mathrm{IS}_{t_{8}}$ & 10000 & 0.034 & 25000 & 0.035 & 50000 & 0.024 \\
$\mathrm{IS}_{\text {mix }}$ & 10000 & 0.030 & 25000 & 0.020 & 50000 & 0.012 \\
$\mathrm{BS}_{\text {mix }}$ & 10000 & 0.029 & 25000 & 0.018 & 50000 & 0.011 \\
$\mathrm{Chib}$ & 8000 & 0.736 & 20000 & 0.486 & 40000 & 0.312 \\
$\mathrm{PP}$ & $20 \times 1600$ & 2.906 & $20 \times 2150$ & 1.936 & $20 \times 3200$ & 1.547 \\
$\mathrm{HM}$ & 37000 & 5.548 & 50000 & 5.331 & 72000 & 4.850 \\
\hline \hline
\end{tabular}

Table 1: Monte Carlo standard errors of log marginal likelihood estimates for different number of Markov chain samples. Standard errors are given across 50 replicates for each of the methods. Each of the methods have roughly the same computational cost.

rates, density-dependence in within-household transmission and the amount of missing data.

\subsubsection{Reversible jump MCMC}

Reversible jump MCMC (Green, 1995) provides a framework for constructing MCMC algorithms that can jump between states with different dimensions. This allows the model indicator to be treated as a parameter to be estimated from the data like any other. Although the Bayes factors can be calculated from the posterior probabilities in favour of each model, the marginal likelihoods themselves cannot be obtained. The main difficulty with RJMCMC lies in designing efficient proposals to jump between models and their associated parameters.

Here we wish to compare model $\mathcal{M}_{1}$ described in Section 5.1 with the nested model $\mathcal{M}_{2}$, in which the community acquisition rates for adults and children are equal, i.e. $k_{1}=k_{2}=k$ for some $k$ (Section 5.5). When in model $\mathcal{M}_{1}$, we propose a move to $\mathcal{M}_{2}$ with probability 0.5 , in which the joint community acquisition rate $k$ is set to $k=\frac{L_{1} k_{1}+L_{2} k_{2}}{L_{1}+L_{2}}$, where $L_{1}$ is the total number of children and $L_{2}$ is the total number of adults. The Jacobian of the transformation is $\frac{L_{1} L_{2}}{L_{1}+L_{2}}$. For the reverse move, we need to increase the 
dimension of the parameter vector, therefore an auxiliary random variable $U$ is required. Let $U \sim N\left(0, \sigma^{2}\right)$ with $\sigma^{2}$ fixed but well chosen. Then we set $k_{1}=k+\frac{u}{L_{1}}$ and $k_{2}=k-\frac{u}{L_{2}}$. The Jacobian of the transformation is then $\frac{L_{1}+L_{2}}{L_{1} L_{2}}$. The acceptance probability of jumping from $\mathcal{M}_{1}$ to $\mathcal{M}_{2}$, is given by $\min \left(1, A_{12}\right)$ where

$$
A_{12}=\frac{\pi\left(\mathcal{M}_{2}, \phi_{2} \mid \mathbf{x}\right) \pi\left(\mathcal{M}_{2}\right)}{\pi\left(\mathcal{M}_{1}, \phi_{1} \mid \mathbf{x}\right) \pi\left(\mathcal{M}_{1}\right)}\left(\frac{1}{\sigma \sqrt{2 \pi}} e^{-\frac{1}{2 \sigma^{2}}\left(\frac{L_{1} L_{2}\left(k_{1}-k_{2}\right)}{L_{1}+L_{2}}\right)^{2}}\right) \frac{L_{1} L_{2}}{L_{1}+L_{2}},
$$

where $\phi_{1}=\left(k_{1}, k_{2}, \beta_{11}, \beta_{12}, \beta_{21}, \beta_{22}, w, \mu_{1}, \mu_{2}, \pi_{1}, \pi_{2}, \mathbf{y}\right)$ and $\phi_{2}=\left(k, \beta_{11}\right.$, $\left.\beta_{12}, \beta_{21}, \beta_{22}, w, \mu_{1}, \mu_{2}, \pi_{1}, \pi_{2}, \mathbf{y}\right)$. For the reciprocal move from model $\mathcal{M}_{2}$ to $\mathcal{M}_{1}$, the probability of accepting the jump is given by $\min \left(1, A_{21}\right)$ where

$$
A_{21}=\frac{\pi\left(\mathcal{M}_{1}, \phi_{1} \mid \mathbf{x}\right) \pi\left(\mathcal{M}_{1}\right)}{\pi\left(\mathcal{M}_{2}, \phi_{2} \mid \mathbf{x}\right) \pi\left(\mathcal{M}_{2}\right)}\left(\frac{1}{\sigma \sqrt{2 \pi}} e^{-\frac{u^{2}}{2 \sigma^{2}}}\right)^{-1} \frac{L_{1}+L_{2}}{L_{1} L_{2}} .
$$

In addition to the model-switching step, the within-model parameters are updated using a standard MCMC algorithm that employs both Gibbs sampler updates and random walk Metropolis steps with a Gaussian proposal density centred at the current value.

\subsubsection{Heterogeneity in household transmission rates}

We wish to evaluate whether or not there is heterogeneity in the household transmission rates. More precisely, we wish to compare the full model $\mathcal{M}_{1}$ with the special case in which the within-household acquisition rates are identical between the two age groups, i.e. $\beta_{11}=\beta_{12}=\beta_{21}=\beta_{22}=\beta$ (say), which we call model $\mathcal{M}_{3}$. This kind of question is extremely challenging to answer using reversible jump methodology because it is difficult to move efficiently between models when this involves a large change in dimension. Again we generated two datasets, one from $\mathcal{M}_{1}$ using the parameters given in Section 5.4, and one from $\mathcal{M}_{3}$ with $\beta=0.0515$, the average of $\beta_{11}, \beta_{12}, \beta_{21}$ and $\beta_{22}$. For both datasets, we calculated Bayes factors using importance sampling, bridge sampling, Chib's method, power posteriors and the harmonic mean. Our objective was to check that the correct model was chosen by the Bayes factors criterion in this setting.

Table 2 presents the marginal likelihood estimates and the corresponding Monte Carlo standard errors for each method, where bold entries show the preferred model. The importance sampling, bridge sampling, Chib and power posterior methods all agreed and were able to discriminate the true model. The estimates of the log marginal likelihoods are similar within Monte Carlo error, with importance and bridge sampling being the most 
precise. As was previously observed in Section 5.4, the harmonic mean overestimated the log marginal likelihoods and yielded inaccurate results, favouring the wrong model in both scenarios.

\begin{tabular}{|c|c|c|c|c|}
\hline $\begin{array}{c}\text { Simulation } \\
\text { designs }\end{array}$ & Method & $\begin{array}{l}\text { Log marginal of } \\
\text { model } \mathcal{M}_{1}\end{array}$ & $\begin{array}{l}\text { Log marginal of } \\
\text { model } \mathcal{M}_{3}\end{array}$ & $\log B_{13}$ \\
\hline \multirow{5}{*}{$\begin{array}{c}\text { Data } \\
\text { from } \mathcal{M}_{1}\end{array}$} & $\mathrm{IS}_{\operatorname{mix}}$ & $\mathbf{- 1 2 6 7 . 1 0 2}(0.018)$ & $-1268.843(0.020)$ & $1.742(0.031)$ \\
\hline & $\mathrm{BS}_{\operatorname{mix}}$ & $\mathbf{- 1 2 6 7 . 1 0 4}(0.018)$ & $-1268.844(0.019)$ & $1.744(0.029)$ \\
\hline & Chib & $\mathbf{- 1 2 6 6 . 9 9 9}(0.261)$ & $-1268.075(0.619)$ & $1.190(0.729)$ \\
\hline & $\mathrm{PP}$ & $\mathbf{- 1 2 6 2 . 9 5 7}(1.926)$ & $-1266.150(2.107)$ & $3.215(2.465)$ \\
\hline & $\mathrm{HM}$ & $-931.320(3.882)$ & $\mathbf{- 9 2 9 . 1 6 8}(5.444)$ & $-3.562(6.507)$ \\
\hline \multirow{5}{*}{$\begin{array}{c}\text { Data } \\
\text { from } \mathcal{M}_{3}\end{array}$} & $\mathrm{IS}_{\text {mix }}$ & $-1512.107(0.011)$ & $\mathbf{- 1 5 0 5 . 0 5 8}(0.015)$ & $-7.048(0.019)$ \\
\hline & $\mathrm{BS}_{\text {mix }}$ & $-1512.101(0.011)$ & $\mathbf{- 1 5 0 5 . 0 6 0}(0.013)$ & $-7.042(0.021)$ \\
\hline & Chib & $-1512.110(0.326)$ & $\mathbf{- 1 5 0 5 . 0 2 1}(0.290)$ & $-7.156(0.445)$ \\
\hline & $\mathrm{PP}$ & $-1509.138(2.003)$ & $\mathbf{- 1 5 0 0 . 6 1 6}(2.089)$ & $-8.833(2.495)$ \\
\hline & $\mathrm{HM}$ & $\mathbf{- 1 1 8 4 . 7 5 5}(5.150)$ & $-1195.668(6.252)$ & $9.273(7.552)$ \\
\hline
\end{tabular}

Table 2: Bayes factors and log marginal likelihoods of the main and reduced models for the two simulation designs. The Monte Carlo standard errors over 50 replicates are shown in parentheses.

\subsubsection{Density-dependence in within-household transmission}

Melegaro et al. (2004) investigated the relationship between transmission rates and household size via the density correction factor $(z-1)^{w}$ in the transmission rates, Equation (16) in the main text, where $z$ is the household size. Since their confidence interval for $w$ included 1 they were unable to determine whether transmission increased $(w<1)$ or decreased $(w>1)$ with household size. Moreover, the value $w=1$ corresponds to frequency dependent transmission, where the average number of contacts is the same irrespective of household size. We wish to determine whether frequency dependent transmission $\left(w=1\right.$, which we call model $\left.\mathcal{M}_{4}\right)$ could be identified from the data.

Bayesian model comparison problems of this kind often suffer from Lindley's paradox, where the choice of prior for $w$ in the more complex model has undue influence on the resulting Bayes factor. To reduce (but not remove) the impact of Lindley's paradox we consider two priors for $w$ in $\mathcal{M}_{1}$ : Gamma(1,1) (referred as the local prior) and the inverse moment prior for 
$\log w$ (referred as the non local prior), with densities respectively given by

$$
\begin{aligned}
\pi_{L}(w) & =\frac{b^{a} w^{a-1} e^{-w b}}{\Gamma(a)}, \quad a=1, b=1 \\
\pi_{N L}(w) & =\frac{\kappa \tau^{\nu / 2}}{w \Gamma(\nu / 2 \kappa)}(\log (w))^{-(\nu+1)} \exp \left[-\left\{\frac{(\log (w))^{2}}{\tau}\right\}^{-\kappa}\right],
\end{aligned}
$$

with $\kappa=1, \nu=1$ and $\tau=0.173$ (for more details see Johnson and Rossell (2010)). The density functions of the two priors are shown in Figure 5. The figure illustrates the fact that the non local prior has density zero at $w=1$.

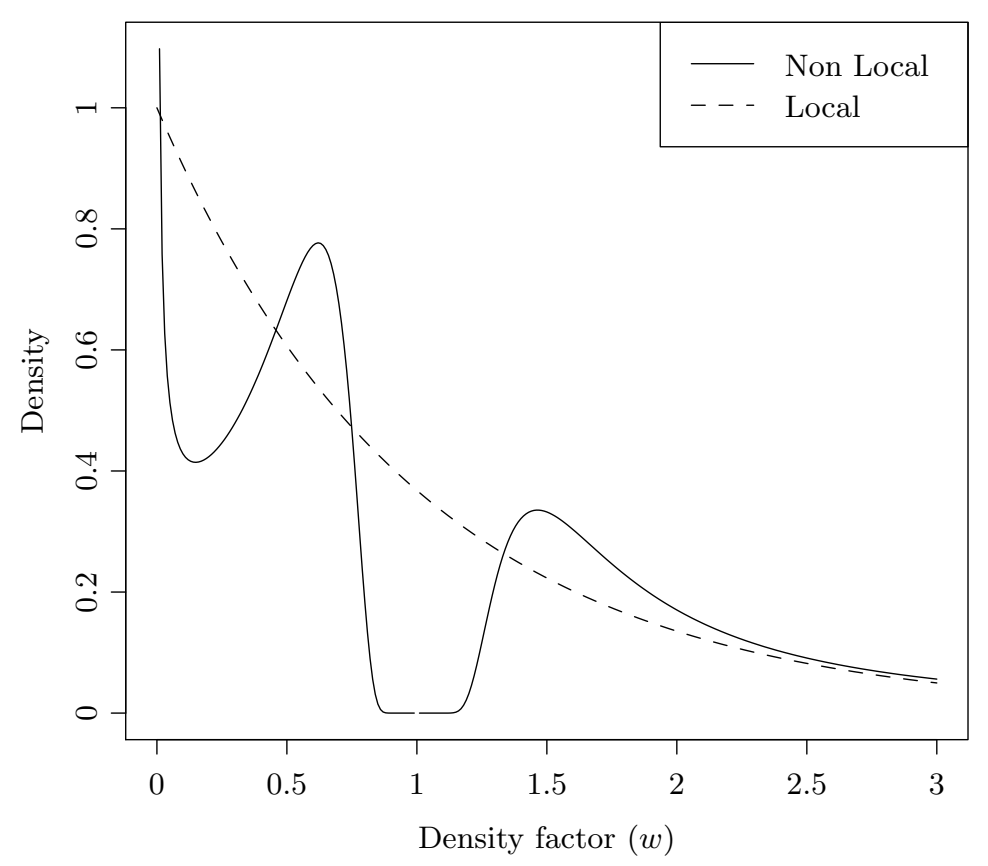

Figure 5: Prior densities on the density correction factor $w$.

To determine if evidence in favour or against $\mathcal{M}_{4}$ could be determined from the study of Melegaro et al. (2004) we simulated datasets of equivalent size with values for $w$ from 0.5 through to 2 , increasing by 0.1 each time. For each value of $w$ we obtained an estimate of the posterior probability of $\mathcal{M}_{1}$ along with its standard error, based on 100 simulated datasets. Results are shown in Figure 6. For values of $w$ close to 1, the non local prior provided on average stronger evidence in favour of the simple model even though model $\mathcal{M}_{1}$ was technically the correct model. For values of $w$ within the interval $[0.6,1.4]$ both priors supported $\mathcal{M}_{4}$, but only the non local prior provided positive support for $\mathcal{M}_{4}$. Whereas when $w$ went from 1.5 to 2 , both priors favoured $\mathcal{M}_{1}$, with the non local prior providing equal or higher posterior 
probability in favour of the correct model than the alternative local prior. Melegaro et al. (2004) estimated $w=1.18$ and in this region we expect weak support for frequency dependent transmission, model $\mathcal{M}_{4}$.

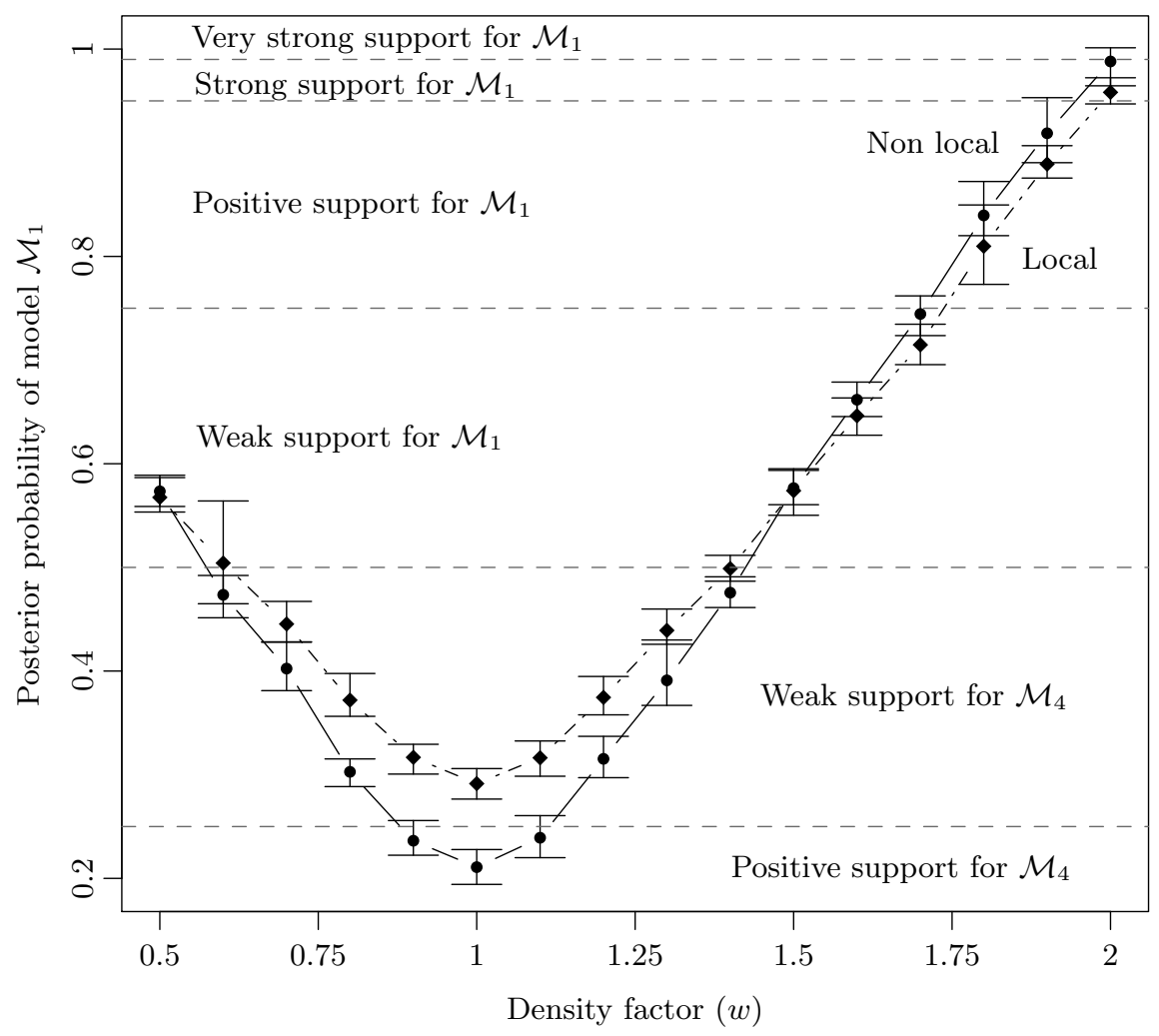

Figure 6: Posterior probability of the full model using two different prior specifications; the local prior $(-\cdot-\cdot-)$ and the non local prior ( - ). Error bars represent the Monte Carlo standard error based on 100 simulations.

\subsubsection{Amount of missing data}

In this section we wish to assess the accuracy and efficiency of the proposed method as a function of the total number of hidden states. One way to vary the amount of missing data without diluting the information content of the dataset is to vary the time interval $\delta t$. The larger $\delta t$ is, the smaller the number of hidden states that need to be imputed. For example, when $\delta t=1$ 60840 hidden states need to be imputed, whereas we have only 3900 when $\delta t=10$. For $\delta t=1,2, \ldots, 10$, we generated 10 synthetic datasets according to $\mathcal{M}_{1}$. For each dataset we fitted 10 different models, one for each possible 
value of $\delta t$, and calculated the log marginal likelihood.

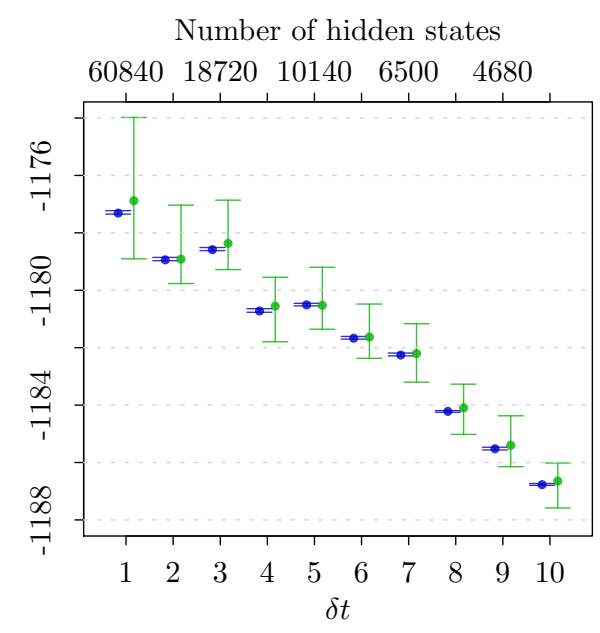

(a) Data generated from $\delta t=1$.

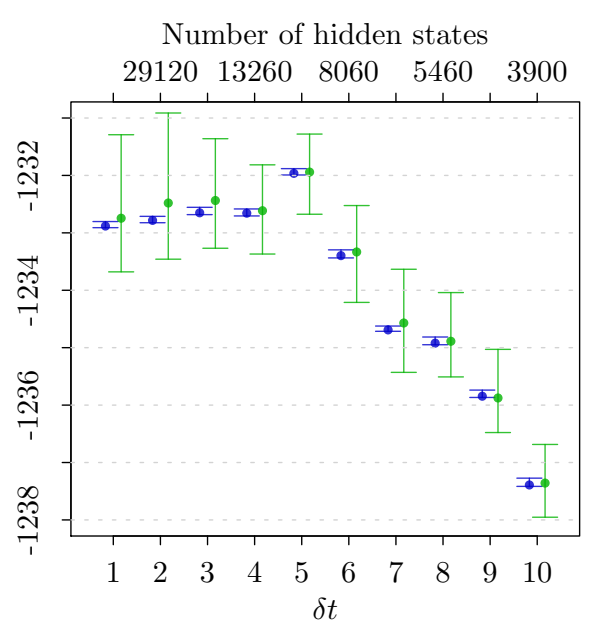

(b) Data generated from $\delta t=5$.

Number of hidden states

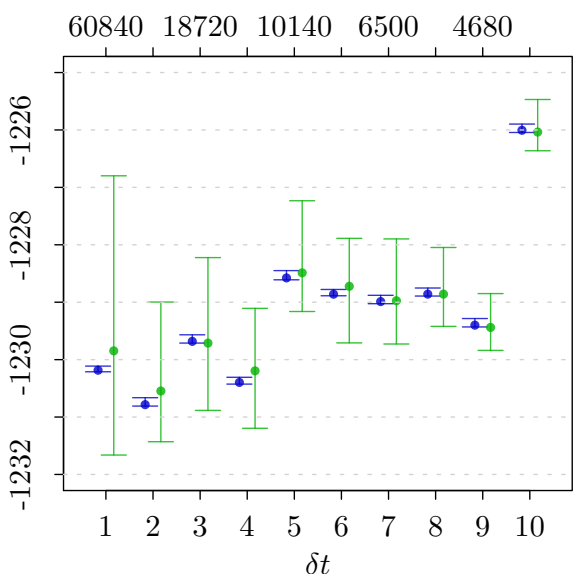

(c) Data generated from $\delta t=10$.

Figure 7: Sensitivity to $\delta t$, the time interval. Log marginal likelihood estimation and its corresponding $95 \%$ credible interval using data generated by (a) $\delta t=1$, (b) $\delta t=5$ and (c) $\delta t=10$.

For brevity, Figure 7 presents results only for the data generated by $\delta t=$ $1,5,10$. In all three cases, the log marginal likelihood curves are peaked at the true value of $\delta t$, the one used to create the data (Figure 7). The marginal likelihood estimates from Chib's method are also maximized at true values, but since the standard errors are much higher, more samples would be required to distinguish between the competing models.

Figure 8 shows how the Monte Carlo standard errors in Chib's method and the importance sampling method increase as a function of the total number 


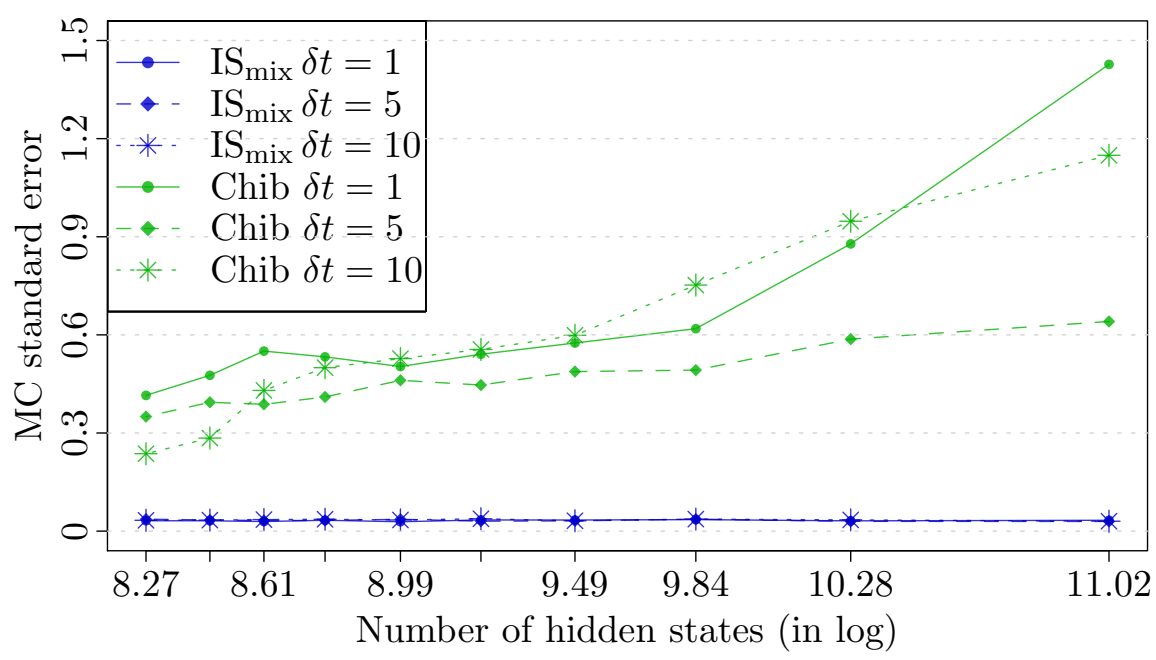

Figure 8: Monte Carlo standard error of the proposed importance sampling and Chib's methods for different number of hidden states and values of time interval $\delta t$.

of hidden states. The graph shows that the Monte Carlo standard errors from the importance sampling method appear very stable as the dimensionality of the hidden states is increase.

\section{References}

Ball, F.G., Mollison, D. and Scalia-Tomba, G. (1997) Epidemics with two levels of mixing. Ann. Appl. Prob. 7, 46-89.

Chib, S. (1995) Marginal likelihood from the Gibbs output. Journal of the American Statistical Association 90, 1313-1321.

Chib, S. and Jeliazkov, I. (2001) Marginal likelihood from the MetropolisHastings output. Journal of the American Statistical Association 96, 270281.

Friel, N. and Pettitt, A. N. (2008) Marginal likelihood estimation via power posteriors. Journal of the Royal Statistical Society: Series B (Statistical Methodology) 70, 589-607.

Green P.J. (1995). Reversible jump Markov chain Monte Carlo computation and Bayesian model determination. Biometrika 82, 711-732.

Melegaro, A., Gay, N., and Medley, G. (2004) Estimating the transmission 
parameters of pneumococcal carriage in households. Epidemiology and Infection 132, 433-441.

Meng, X-L. and Wong, W.H. (1996) Simulating ratios of normalizing constants via a simple identity: A theoretical exploration. Statistica Sinica 6, 831-860.

Neal, P. and Kypraios, T. (2015) Exact Bayesian inference via data augmentation. Stats. and Computing 25, 333-347

Newton, M.A. and Raftery, A.E. (1994) Approximate Bayesian inference with the weighted likelihood bootstrap. Journal of the Royal Statistical Society: Series B (Statistical Methodology) 56, 3-48.

Johnson, V. E. and Rossell, D. (2010) On the use of non-local prior densities in Bayesian hypothesis tests. Journal of the Royal Statistical Society: Series B (Statistical Methodology) 72, 143-170. 